\title{
Severe Hypoglycemia-induced Right Hemiparesis with Reversible Diffusion Restriction in the Left Internal Capsule Due to Combination Therapy Using Disopyramide and Clarithromycin
}

\author{
Yoshito Sugita, ${ }^{1}$ Masaomi Koyanagi, ${ }^{1}$ Masashi Oda, ${ }^{1}$ Tamaki Kobayashi, ${ }^{1}$ Osamu Narumi, ${ }^{1}$ and Masaaki Saiki ${ }^{1}$
}

\begin{abstract}
Severe hypoglycemia is known to cause acute focal neurological symptoms. In cases with a medical history of diabetes mellitus (DM), the diagnosis and treatment of hypoglycemia-induced neurological symptoms are simple. However, severe hypoglycemia can occur in patients who are not taking hypoglycemic agents such as insulin or longacting sulfonylurea drugs. We describe a 95-year-old man with sudden onset of right hemiparesis who showed high signal intensity on diffusion-weighted imaging involving the left internal capsule with corresponding reduced apparent diffusion coefficient hypointensity. Laboratory findings revealed severe hypoglycemia $(27 \mathrm{mg} / \mathrm{dl})$. However, he was not taking insulin or long-acting sulfonylurea drugs but disopyramide and clarithromycin had been administered. In addition, he had kidney dysfunction with an estimated glomerular filtration rate (GFR) of $42.9 \mathrm{ml} /$ $\mathrm{min} / 1.73 \mathrm{~m}^{2}$. After the blood glucose level was normalized, the left hemiparesis completely recovered and abnormal findings of magnetic resonance imaging (MRI) study also became normal. A combination of disopyramide and clarithromycin may cause severe hypoglycemia-induced neurological symptoms particularly in patients with kidney dysfunction. Even in a patient with sudden-onset hemiparesis and no history of DM, the possibility of hypoglycemiainduced neurological deficit should be considered.
\end{abstract}

Keywords: hypoglycemia, disopyramide, clarithromycin, diffusion MRI, metabolism

\section{Introduction}

Severe hypoglycemia is known to cause acute focal neurological symptoms, such as hemiparesis, with findings similar to acute ischemic stroke on magnetic resonance imaging (MRI). ${ }^{1,2)}$ In cases with a medical history of diabetes mellitus (DM), the diagnosis and treatment of hypoglycemia-induced neurological deficit are simple. However, if there is no history of DM, it is common for patients with such symptoms to be initially diagnosed with and treated for acute ischemic stroke.

${ }^{1}$ Department of Neurosurgery, National Hospital Organization Himeji Medical Center, Himeji, Hyogo, Japan

Received: February 19, 2017; Accepted: June 22, 2017

Online November 21, 2017

Copyright $₫ 2018$ by The Japan Neurosurgical Society

This work is licensed under a Creative Commons AttributionNonCommercial-NoDerivatives International License.
Here, we present a case of right hemiparesis, severe hypoglycemia, and diffusion restriction and corresponding apparent diffusion coefficient (ADC) hypointensity in the left internal capsule on MRI in a patient without a history of DM. Normalization of the blood glucose levels resulted in the complete recovery of the neurological and radiological findings. In patients with acute neurological impairment without a history of DM, we should consider the possibility of hypoglycemia-induced neurological impairment and focus on drugs that could cause hypoglycemia.

\section{Case Report}

A 95-year-old man presented to our hospital with sudden onset of right hemiparesis (manual muscle testing: 1/5) and minor disturbance in consciousness (Glasgow Coma Scale: 13/15). He had a medical history of ventricular tachycardia and pneumonia but no history of DM, hepatitis, or gastrectomy, which have a potential risk for causing hypoglycemia. He was orally administered with $300 \mathrm{mg}$ t.i.d. disopyramide for tachycardia and clarithromycin and $200 \mathrm{mg}$ b.i.d. disopyramide for pneumonia. Emergent MRI demonstrated high signal intensity on diffusion-weighted imaging (DWI) involving the left internal capsule with corresponding reduced ADC (Fig. 1).

The initial diagnosis appeared to be an acute stroke. However, laboratory examination revealed a low blood glucose level $(27 \mathrm{mg} / \mathrm{dl})$. On intravenous administration of $20 \mathrm{ml}$ of $50 \%$ glucose, the blood glucose level was normalized, and the right hemiparesis immediately and completely recovered.

Follow-up DWI sequences, taken $18 \mathrm{~h}$ after the initial MRI, showed prominent regression of the hyperintense lesion and corresponding ADC hypointensity (Fig. 2). The patient was discharged from the hospital without any neurological deficit.

\section{Discussion}

In patients with symptomatic hypoglycemia mimicking an acute stroke, imaging abnormalities on computed tomography (CT) and MRI were seen in 20\%. ${ }^{3)}$ For imaging, DWI sequences on MRI are the most sensitive for detecting lesions with restricted diffusion with a corresponding ADC hypointensity, associated with hypoglycemia-induced neurological deficits. ${ }^{4)}$ In comatose patients with hypoglycemia, hyperintensity lesions on DWI, corresponding to hypointense signals on the ADC map, were seen in 9\%-64\%., ${ }^{5,6)}$ Of these, the posterior limb of the internal capsule is one of the most affected areas in the early period. 

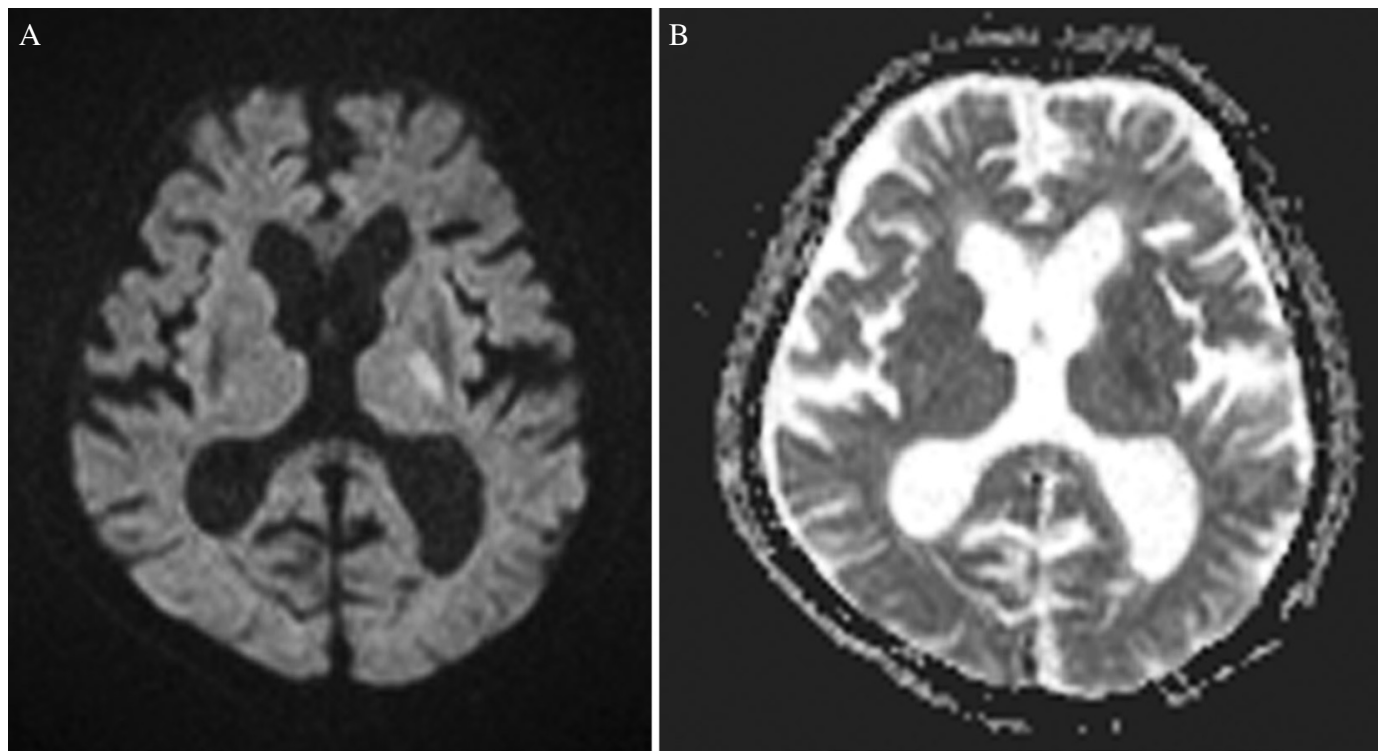

Fig. 1 High-intensity signals on diffusion-weighted imaging revealed the left internal capsule (A) and reduced apparent diffusion coefficient (B).
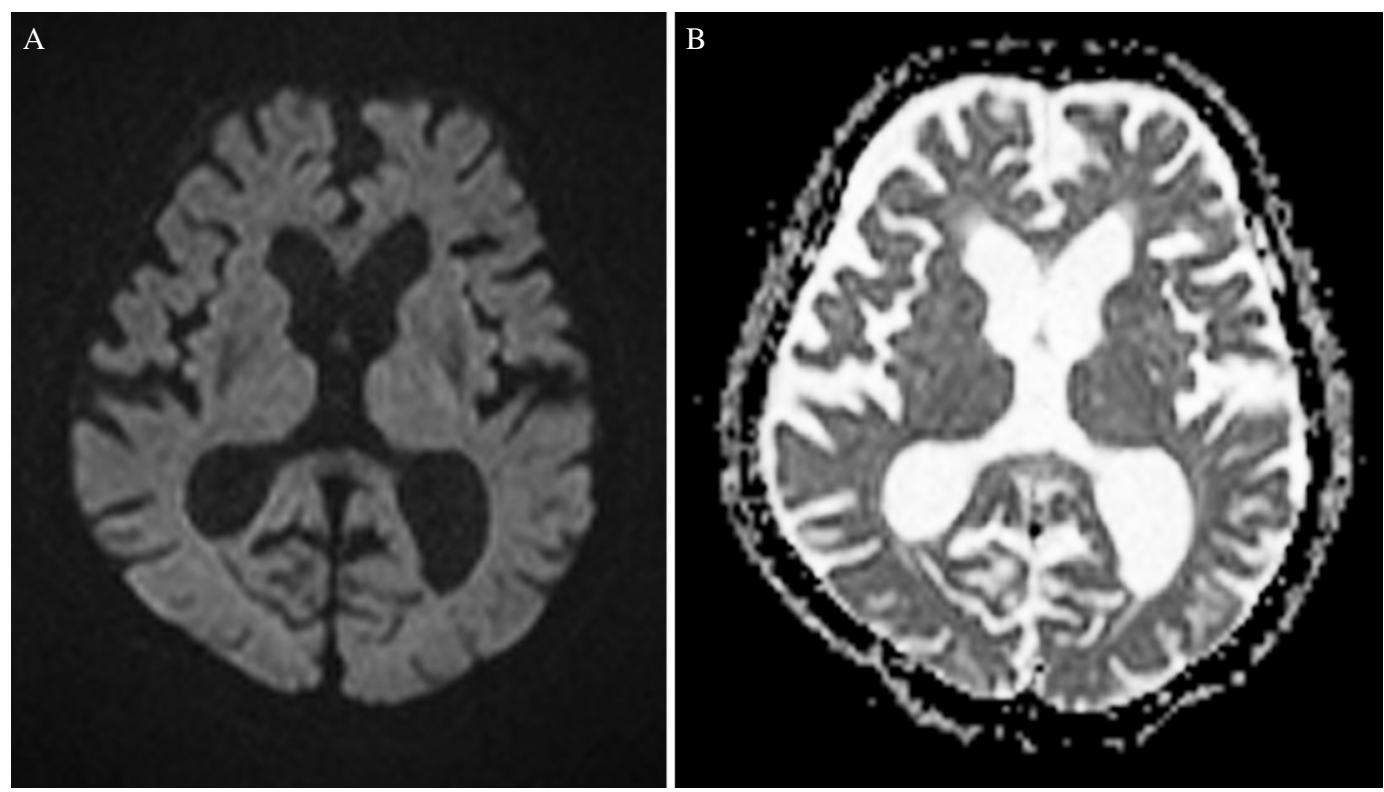

Fig. 2 Follow-up diffusion-weighted imaging post $18 \mathrm{~h}$ (A) and apparent diffusion coefficient hypointensity (B) showed prominent regression of the hyperintense lesion.

Hypoglycemic brain damage often takes an asymmetrical pattern. Approximately, half of the lesions were ipsilateral. ${ }^{5)}$ In this case, DWI high-signal intensity was seen only in the left internal capsule. The underlying mechanism of this asymmetric distribution pattern of high-intensity signals on DWI and an accompanying reduction in ADC values in patients with hypoglycemia remains unclear. Because a reduction in ADC values follows the establishment of cerebral isoelectricity, ${ }^{5,7,8)}$ the asynchronous onset of isoelectricity between the hemispheres could explain this phenomenon.9)

The development of hemiplegia with lesions of the contralateral internal capsule may be initially diagnosed and treated as acute ischemic stroke. Delayed diagnosis can potentially increase morbidity and mortality; therefore, the possibility of hypoglycemia-induced neurological deficit must be kept in mind. Blood glucose testing should be performed in patients, who present with acute neurological impairment. ${ }^{3,6)}$

Hypoglycemia is common in patients with diabetes and can occur as a complication of insulin or long-acting sulfonylurea therapy. In these situations, the diagnosis of hypoglycemia-induced neurological symptoms is simple. Our patient had severe hypoglycemia without a medical history of DM. However, he was being treated with disopyramide for ventricular tachycardia. Disopyramide is a sodium channel blocker and can cause hypoglycemia by enhancing 
insulin secretion from pancreatic beta cells through sodium channel inhibition. ${ }^{10)}$

In addition, the patient had kidney dysfunction with an estimated glomerular filtration rate (GFR) of $42.9 \mathrm{ml} / \mathrm{min} /$ $1.73 \mathrm{~m}^{2}$. Because approximately half of any given dose of disopyramide is excreted in the urine, in a patient with kidney dysfunction, the blood level of disopyramide can increase. Furthermore, the patient was also taking clarithromycin, which is known to suppress CYP3A4. Because CYP3A4 metabolizes disopyramide, disopyramide combined with clarithromycin can reduce disopyramide metabolism. Through these mechanisms, the blood glucose lowering effect of disopyramide can be enhanced and can result in severe hypoglycemia. ${ }^{11)}$

Drugs are the most common cause of hypoglycemia often in the setting of chronic kidney disease (CKD) among the causes of hypoglycemia in patients without DM. ${ }^{12)}$ Many drugs, in addition to insulin, such as long-acting sulfonylurea drugs and alcohol can cause hypoglycemia. It is necessary to understand the various drugs, which can cause hypoglycemia, such as angiotensin converting enzyme inhibitors, angiotensin receptor antagonists, levofloxacin, trimethoprimsulfamethoxazole, heparin, and 6-mercaptopurine. ${ }^{12)}$

In conclusion, even in a patient with emergent hemiparesis and no history of DM, the possibility of hypoglycemia-induced neurological deficit should be considered. Hypoglycemia can occur in patients who are not taking hypoglycemic agents such as insulin or long-acting sulfonylurea drugs. A combination of disopyramide and clarithromycin may cause severe hypoglycemia, particularly in patients with CKD.

\section{Conflicts of Interest Disclosure}

The authors declare no conflicts of interest. All authors who are members of The Japan Neurosurgical Society (JNS) have registered online Self-reported COI Disclosure Statement Forms through the website for JNS members.

\section{References}

1) Albayram S, Ozer H, Gokdemir S, et al.: Reversible reduction of apparent diffusion coefficient values in bilateral internal capsules in transient hypoglycemia-induced hemiparesis. AJNR Am J Neuroradiol 27: 1760-1762, 2006

2) Aoki T, Sato T, Hasegawa K, Ishizaki R, Saiki M: Reversible hyperintensity lesion on diffusion-weighted MRI in hypoglycemic coma. Neurology 63: 392-393, 2004

3) Yong AW, Morris Z, Shuler K, Smith C, Wardlaw J: Acute symptomatic hypoglycaemia mimicking ischaemic stroke on imaging: a systemic review. BMC Neurol 12: 139, 2012

4) Böttcher J, Kunze A, Kurrat C, et al.: Localized reversible reduction of apparent diffusion coefficient in transient hypoglycemia-induced hemiparesis. Stroke 36: e20-e22, 2005

5) Johkura K, Nakae Y, Kudo Y, Yoshida TN, Kuroiwa Y: Early diffusion MR imaging findings and short-term outcome in comatose patients with hypoglycemia. AJNR Am J Neuroradiol 33: 904-909, 2012

6) Katoh M, Yoshino M, Aoki T, Abumiya T, Imamura H, Aida T: Localized reversible high signal intensities on diffusion-weighted MRI in hypoglycemia: a study of 70 cases. Asian J Neurosurg 11: 412-415, 2016

7) Schmidt P, Böttcher J, Ragoschke-Schumm A, et al.: Diffusionweighted imaging of hyperacute cerebral hypoglycemia. AJNR Am J Neuroradiol 32: 1321-1327, 2011

8) Hasegawa Y, Formato JE, Latour LL, et al.: Severe transient hypoglycemia causes reversible change in the apparent diffusion coefficient of water. Stroke 27: 1648-1655; discussion 1655-1656, 1996

9) Auer RN: Hypoglycemic brain damage. Forensic Sci Int 146: 105-110, 2004

10) Abe M, Maruyama T, Fujii Y, et al.: Disopyramide-induced hypoglycemia in a non-diabetic hemodialysis patient: a case report and review of the literature. Clin Nephrol 76: 401-406, 2011

11) Iida H, Morita T, Suzuki E, Iwasawa K, Toyo-oka T, Nakajima T: Hypoglycemia induced by interaction between clarithromycin and disopyramide. Jpn Heart J 40: 91-96, 1999

12) Cryer PE, Axelrod L, Grossman AB, et al.; Endocrine Society: Evaluation and management of adult hypoglycemic disorders: an endocrine society clinical practice guideline. J Clin Endocrinol Metab 94: 709-728, 2009

Corresponding author: Masaomi Koyanagi, MD, PhD, Department of Neurosurgery, National Hospital Organization Himeji Medical Center, 68 Honnmachi, Himeji, Hyogo 670-8520, Japan

$\triangle$ koyanagm@gmail.com 